\title{
Erratum to: Final overall survival in JO22903, a phase II, open-label study of first-line erlotinib for Japanese patients with EGFR mutation-positive non-small-cell lung cancer
}

\author{
Noboru Yamamoto ${ }^{1}$ Koichi Goto ${ }^{2} \cdot$ Makoto Nishio $^{3} \cdot$ Kenichi Chikamori $^{4}$. \\ Toyoaki Hida $^{5} \cdot$ Makoto Maemondo $^{6} \cdot$ Nobuyuki Katakami $^{7} \cdot$ Toshiyuki Kozuki $^{8}$. \\ Hiroshige Yoshioka ${ }^{9}$ Takashi Seto $^{10} \cdot$ Kosei Tajima $^{11} \cdot$ Tomohide Tamura $^{12}$
}

Published online: 17 October 2016

(C) Japan Society of Clinical Oncology 2016

\section{Erratum to: Int J Clin Oncol DOI 10.1007/s10147-016-1039-0}

In the original publication of the article, Table 4 was published incorrectly. The corrected Table 4 is published with this erratum.
Table 4 Treatment-related adverse events, all grades $(\geq 30 \%)$ and grade $\geq 3$

\begin{tabular}{llc}
\hline & All grades $(\geq 30 \%)$ & Grade $\geq 3$ \\
\hline Rash & $85(82.5)$ & $15(14.6)$ \\
Diarrhea & $82(79.6)$ & $1(1.0)$ \\
Dry skin & $82(79.6)$ & $5(4.9)$ \\
Paronychia & $69(67.0)$ & $1(1.0)$ \\
Stomatitis & $65(63.1)$ & $1(1.0)$ \\
Pruritus & $67(65.0)$ & $3(2.9)$ \\
Decreased appetite & $35(34.0)$ & $2(1.9)$ \\
ALT increased & $33(32.0)$ & $9(8.7)$ \\
\hline
\end{tabular}

$A L T$ alanine aminotransferase

7 Integrated Oncology, Institute of Biomedical Research and Innovation Hospital, Kobe, Hyogo, Japan

8 Department of Thoracic Oncology, National Hospital Organization, Shikoku Cancer Centre, Matsuyama, Ehime, Japan

9 Department of Respiratory Medicine, Kurashiki Central Hospital, Kurashiki, Okayama, Japan

10 Department of Thoracic Oncology, National Kyushu Cancer Centre, Fukuoka, Fukuoka, Japan

11 Clinical Research Planning Department, Chugai Pharmaceutical Co. Ltd, Chuo-ku, Tokyo, Japan

12 Thoracic Center, St Luke's International Hospital, Chuo-ku, Tokyo, Japan

5 Department of Thoracic Oncology, Aichi Cancer Centre Hospital, Nagoya, Aichi, Japan

6 Department of Respiratory Medicine, Miyagi Cancer Centre, Natori, Miyagi, Japan 